EPJ Web of Conferences 73, 02019 (2014)

DOI: $10.1051 /$ epjconf/20147302019

(C) Owned by the authors, published by EDP Sciences, 2014

\title{
Flavor decomposition of transverse momentum dependent parton distributions
}

\author{
Alessio Del Dotto ${ }^{1}$, Leonid Kaptari ${ }^{2}$, Emanuele Pace ${ }^{3}$, Giovanni Salmè ${ }^{4}$, Sergio Scopetta ${ }^{5}$ \\ and Evaristo Cisbani ${ }^{6}$ \\ ${ }^{1}$ Università di Roma Tre and INFN, Roma 3, Italy \\ ${ }^{2}$ Bogoliubov LTP, JINR, Dubna, Russia \\ ${ }^{3}$ Università di Roma "Tor Vergata" and INFN, Roma 2, Italy \\ ${ }^{4}$ INFN, Sezione di Roma, Italy \\ ${ }^{5}$ Università di Perugia and INFN, Sezione di Perugia, Italy \\ ${ }^{6}$ INFN, Sezione di Roma, Gruppo Collegato Sanità and Istituto Superiore di Sanità, Italy
}

\begin{abstract}
We present an improved description of the semi-inclusive deep inelastic electron scattering off polarized ${ }^{3} \mathrm{He}$, providing information on the neutron single spin asymmetries. The analysis at finite momentum transfers in a Poincaré covariant framework is outlined and a quantitative estimate of the nuclear effects is presented.
\end{abstract}

\section{Introduction}

In the last decade considerable experimental and theoretical efforts were carried out to extract the transverse momentum dependent distribution functions (TMDs). Evidence of flavor dependence of unpolarized TMDs has been shown and high precision measurements are needed to disentangle the different flavor contributions [1]. Along this path, high luminosity SiDIS experiments by a polarized ${ }^{3}$ He target will take place at the Thomas Jefferson National Accelerator Facility (JLab) [2]. Our aim is to extend the previous analysis, that addressed the extractions of the neutron single-spin asymmetries (SSAs) within a plane wave impulse approximation (PWIA) framework and in the Bjorken limit, in order to carefully evaluate the nuclear structure effects in the extraction of neutron TMDs.

\section{Polarized ${ }^{3} \mathrm{He}$ nucleus and neutron properties}

A polarized ${ }^{3} \mathrm{He}$ nucleus at a $90 \%$ level is equivalent to a polarized neutron. To disentangle the nucleon structure from the dynamical nuclear effects, one can use the spin-dependent spectral function of ${ }^{3} \mathrm{He}$, $\mathrm{P}_{\sigma, \sigma^{\prime}}(\vec{p}, E)$, (see, e.g. [3]) that allows one to calculate the probability distribution to find a nucleon with given missing energy, three-momentum and polarization inside the nucleus. With this formalism, one can safely extract [4] the neutron longitudinal asymmetry from the corresponding ${ }^{3} \mathrm{He}$ observable, $A_{3}^{\exp }$,

This is an Open Access article distributed under the terms of the Creative Commons Attribution License 4.0, which permits unrestricted use, distribution, and reproduction in any medium, provided the original work is properly cited. 


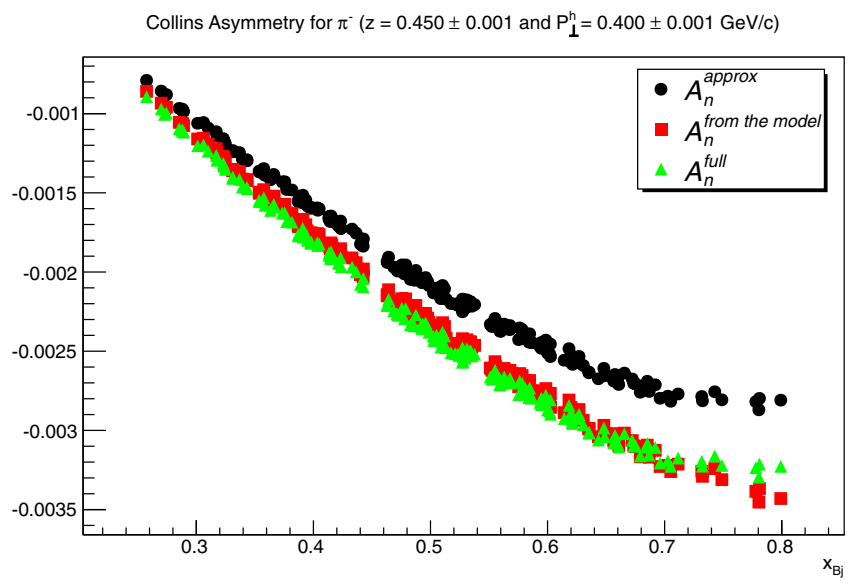

Sivers Asymmetry for $\pi^{-}\left(z=0.450 \pm 0.001\right.$ and $\left.P_{\mathbf{L}}^{h}=0.400 \pm 0.001 \mathrm{GeV} / \mathrm{c}\right)$

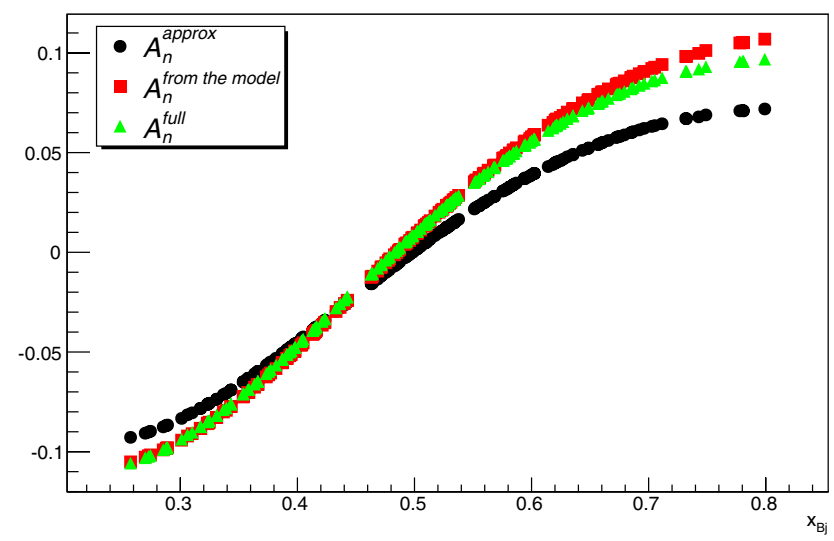

Figure 1. The neutron Collins (upper panel) and Sivers (lower panel) SSAs for the production of $\pi^{-}$vs $x_{B j}$, for $z=0.450 \pm 0.001$ and $P_{\perp}^{h}=0.400 \mathrm{GeV} / \mathrm{c}$, with electron beam energy of $11 \mathrm{GeV}$. Red squares: neutron input model $A_{n}$ [5]. Green triangles: $A_{n}^{\text {full }}$ extracted from Eq. (1). Black dots: $A_{n}^{\text {approx }}$ extracted neglecting the term $2 p_{p} f_{p} A_{p}^{\exp }$.

obtained from the reaction ${ }^{3} \mathrm{He}\left(\vec{e}, e^{\prime}\right) X$ in DIS regime, viz

$$
A_{n} \simeq\left(A_{3}^{\exp }-2 p_{p} f_{p} A_{p}^{\exp }\right) /\left(p_{n} f_{n}\right)
$$

where $p_{n(p)}$ is the neutron (proton) effective polarization inside the polarized ${ }^{3} \mathrm{He}$, and $f_{n(p)}$, the dilution factor. Realistic values of $p_{n}$ and $p_{p}$ are $p_{p}=-0.023, p_{n}=0.878$ (see, e.g., [4, 5]). In [5], an analogous approach was applied to the SSAs of a transversely polarized ${ }^{3} \overrightarrow{\mathrm{He}}$ target (namely Collins and Sivers asymmetries), obtained from the process ${ }^{3} \mathrm{He}\left(e, e^{\prime} \pi\right) X$, in order to extract the SSAs of a transversely polarized neutron. In PWIA and in the Bjorken limit, the SSAs of ${ }^{3} \overrightarrow{\mathrm{He}}$ are a convolution of $\mathrm{P}_{\sigma, \sigma^{\prime}}(\vec{p}, E)$, and the nucleon SSAs, that in turn are convolutions of suitable TMDs and fragmentation functions, that describe the hadronization of the hit quark. This approach has been used in combination with a Monte Carlo code reproducing the experimental conditions and the proper kinematics of [2]. In Fig.1 the nuclear structure effects, based on the previous assumptions are illustrated for a particular choice of the relevant experimental kinematical variables. 


\section{Improvements of the phenomenological description}

To improve the previous description, one can take into account i) relativistic effects in the actual experimental kinematics and ii) the final state interaction (FSI). The relativistic effects [7, 8] will be included within a Poincaré covariant framework for the SSA of ${ }^{3} \mathrm{He}$, based on the Light-Front (LF) Relativistic Hamiltonian Dynamics (RHD), combined with the Bakamijan-Thomas construction of the Poincaré generators. Then, one gets the following convolution for the ${ }^{3} \mathrm{He}$ hadronic tensor $[7,8]$

$$
\mathcal{W}^{\mu \nu}\left(Q^{2}, x_{B}, z, \tau, \hat{\mathbf{h}}, S_{H e}\right) \propto w_{\sigma \sigma^{\prime}}^{\mu \nu}(\tau, \tilde{\mathbf{q}}, \tilde{\mathbf{h}}, \tilde{\mathbf{P}}) \otimes \mathcal{P}_{\sigma^{\prime} \sigma}^{\tau}\left(\tilde{\mathbf{k}}, \epsilon_{S}, S_{H e}\right)
$$

where $w_{\sigma \sigma^{\prime}}^{\mu \nu}(\tau, \tilde{\mathbf{q}}, \tilde{\mathbf{h}}, \tilde{\mathbf{P}})$ is the hadronic nucleon tensor and $\mathcal{P}_{\sigma \sigma^{\prime}}^{\tau}\left(\tilde{\mathbf{k}}, \epsilon_{S}, S_{H e}\right)$ the LF spin-dependent spectral function, related to the instant-form one, $\mathcal{S}_{\sigma_{1}^{\prime} \sigma_{1}}^{\tau}\left(\mathbf{k}, \epsilon_{S}, S_{H e}\right)$, through the unitary Melosh Rotations, $D^{\frac{1}{2}}\left[\mathcal{R}_{M}(\tilde{\mathbf{k}})\right]$, as $\mathcal{P}_{\sigma^{\prime} \sigma}^{\tau}\left(\tilde{\mathbf{k}}, \epsilon_{S}, S_{H e}\right) \propto \sum_{\sigma_{1} \sigma_{1}^{\prime}} D^{\frac{1}{2}}\left[\mathcal{R}_{M}^{\dagger}(\tilde{\mathbf{k}})\right]_{\sigma^{\prime} \sigma_{1}^{\prime}} \mathcal{S}_{\sigma_{1}^{\prime} \sigma_{1}}^{\tau}\left(\mathbf{k}, \epsilon_{S}, S_{H e}\right) D^{\frac{1}{2}}\left[\mathcal{R}_{M}(\tilde{\mathbf{k}})\right]_{\sigma_{1} \sigma}$. In our approach, $\mathcal{S}_{\sigma_{1}^{\prime} \sigma_{1}}^{\tau}$ can be approximated by $\mathrm{P}_{\sigma_{1}^{\prime} \sigma_{1}}^{\tau}$, obtained within a non relativistic framework, since the constraints imposed by the Poincaré covariance can be fully satisfied within our assumptions [6]. The second ingredient to be added is the Generalized Eikonal approximation (GEA) to take care of the FSI effects (see, e.g., [10] and references therein quoted). In particular, the FSI effects to be considered are due to the propagation of the debris, formed after the $\gamma^{*}$ absorption by a target quark, and the subsequent hadronization, both of them influenced by the presence of a fully-interacting $(A-1)$ spectator system. The approximation based on GEA has been recently applied for describing the spectator SiDIS by a polarized ${ }^{3} \mathrm{He}$ target [9]. The key quantities are the intrinsic overlaps defined as follows

$$
\begin{gathered}
\mathcal{O}_{\lambda \lambda^{\prime}}^{\hat{\mathbf{s}}_{A}(F S I)}\left(\mathbf{p}_{N}, E\right)=\sum d \epsilon_{A-1}^{*} \rho\left(\epsilon_{A-1}^{*}\right)\left\langle\hat{S}_{G l}\left\{\Phi_{\epsilon_{A-1}^{*}}, \lambda, \mathbf{p}_{N}\right\} \mid S_{A}, \Phi_{A}\right\rangle\left\langle S_{A}, \Phi_{A} \mid \hat{S}_{G l}\left\{\Phi_{\epsilon_{A-1}^{*}}, \lambda^{\prime}, \mathbf{p}_{N}\right\}\right\rangle \times \\
\delta\left(E+M_{A}-m_{N}-M_{A-1}^{*}-T_{A-1}\right)
\end{gathered}
$$

where i) $E$ is the usual missing energy $E=\epsilon_{A-1}^{*}+B_{A}$, with $\epsilon_{A-1}^{*}\left(\rho\left(\epsilon_{A-1}^{*}\right)\right)$ the energy (state density) of the spectator system and $B_{A}$ the binding energy of the target nucleus, ii) $\mathbf{p}_{N}$ the three-momentum of the struck nucleon and iii) $\hat{S}_{G l}(1,2,3)$ represents the debris-nucleon eikonal scattering S-matrix. A first investigation of FSI was carried out for the spectator SiDIS, where a deuteron is detected in the final state [9]. A relevant outcome of such an analysis was that a wise choice of the kinematical variables can minimize the FSI effects, as already shown in the unpolarized case [10].

\section{Preliminary results on FSI effects and conclusions}

Preliminary insights about FSI effects in the extraction procedure of neutron information can be gained through the comparison, for a JLab kinematics [2], between the PWIA values of both ucleon polarizations and dilution factors that appear in Eq. (1) and the corresponding quantities, $p_{p(n)}^{F S I}, f_{p(n)}^{F S I}$, with FSI effects taken into account. They are related to $\mathcal{P}_{F S I}^{p(n)}\left(\tilde{\mathbf{k}}, \epsilon_{S}, S_{H e}\right)$, the distorted spin-dependent spectral function, defined in terms of the overlaps of Eq. (3) [9], as follows:

$$
\begin{aligned}
& p_{p(n)}^{F S I}=\frac{1}{2} \int d \epsilon_{S} \int d \tilde{\mathbf{k}} \operatorname{Tr}\left[\hat{\mathbf{S}}_{H e} \cdot \sigma \mathcal{P}_{F S I}^{p(n)}\left(\tilde{\mathbf{k}}, \epsilon_{S}, S_{H e}\right)\right], \\
& f_{n,(p)}^{F S I}(x, z)=\frac{\sum_{q} e_{q}^{2} f_{1}^{q, n(p)}(x) D_{1}^{q, h}(z)}{\left\langle N_{n}\right\rangle_{F S I} \sum_{q} e_{q}^{2} f_{1}^{q, n}(x) D_{1}^{q, h}(z)+2\left\langle N_{p}\right\rangle_{F S I} \sum_{q} e_{q}^{2} f_{1}^{q, p}(x) D_{1}^{q, h}(z)} .
\end{aligned}
$$

with $\left\langle N_{n,(p)}\right\rangle_{F S I}=\int d \epsilon_{S} \int d \tilde{\mathbf{k}} \operatorname{Tr}\left[\mathcal{P}_{F S I}^{p(n)}\left(\tilde{\mathbf{k}}, \epsilon_{S}, S_{H e}\right)\right] / 2, f_{1}^{q, n(p)}(x)$ the partonic unpolarized distribution function and $D_{1}^{q, h}(z)$ the unpolarized fragmentation function [5]. 
Table 1.

1) PWIA: $p_{n}=0.878, p_{p}=-0.023, \theta_{e}=30^{\circ}, \theta_{\pi}=14^{\circ}$

\begin{tabular}{|cccccccc|}
\hline $\begin{array}{c}E_{\text {beam }}, \\
\mathrm{GeV}\end{array}$ & $x_{B j}$ & $\begin{array}{c}\nu \\
\mathrm{GeV}\end{array}$ & $\begin{array}{c}p_{\pi} \\
\mathrm{GeV} / \mathrm{c}\end{array}$ & $f_{n}(x, z)$ & $p_{n} f_{n}$ & $f_{p}(x, z)$ & $p_{p} f_{p}$ \\
\hline 8.8 & 0.21 & 7.55 & 3.40 & 0.304 & 0.266 & 0.348 & $-8.410^{-3}$ \\
\hline 8.8 & 0.29 & 7.15 & 3.19 & 0.286 & 0.251 & 0.357 & $-8.510^{-3}$ \\
\hline 8.8 & 0.48 & 6.36 & 2.77 & 0.257 & 0.225 & 0.372 & $-8.910^{-3}$ \\
\hline 11 & 0.21 & 9.68 & 4.29 & 0.302 & 0.265 & 0.349 & $-8.310^{-3}$ \\
\hline 11 & 0.29 & 9.28 & 4.11 & 0.285 & 0.250 & 0.357 & $-8.510^{-3}$ \\
\hline
\end{tabular}

2) FSI: $p_{n}^{F S I}=0.756, p_{p}^{F S I}=-0.027,\left\langle\sigma_{e f f}\right\rangle=71 \mathrm{mb}$

\begin{tabular}{|cccccccc|}
\hline $\begin{array}{c}E_{\text {beam }}, \\
\mathrm{GeV}\end{array}$ & $x_{B j}$ & $\begin{array}{c}\nu \\
\mathrm{GeV}\end{array}$ & $\begin{array}{c}p_{\pi} \\
\mathrm{GeV} / \mathrm{c}\end{array}$ & $f_{n}^{F S T}(x, z)$ & $p_{n}^{\text {FST }} f_{n}^{\text {FST }}$ & $f_{p}^{\text {FST }}(x, z)$ & $p_{p}^{\text {FST }} f_{p}^{\text {FST }}$ \\
\hline 8.8 & 0.21 & 7.55 & 3.40 & 0.353 & 0.267 & 0.405 & $-1.110^{-2}$ \\
\hline 8.8 & 0.29 & 7.15 & 3.19 & 0.332 & 0.251 & 0.415 & $-1.110^{-2}$ \\
\hline 8.8 & 0.48 & 6.36 & 2.77 & 0.298 & 0.225 & 0.432 & $-1.210^{-2}$ \\
\hline 11 & 0.21 & 9.68 & 4.29 & 0.351 & 0.266 & 0.405 & $-1.100^{-2}$ \\
\hline 11 & 0.29 & 9.28 & 4.11 & 0.331 & 0.250 & 0.415 & $-1.110^{-2}$ \\
\hline
\end{tabular}

As shown in Table 1, FSI can sizably modify the overlaps given in Eq. (3), with respect to the PWIA values. In particular, about a $15-20 \%$ depolarization effect of the nucleons in ${ }^{3} \mathrm{He}$ is produced. However it has to be pointed that, even if both polarizations and dilution factors in Eq. (1) are affected by the presence of FSI, fortunately their product does not. Therefore the extraction procedure, can be safely applied and carefully tested in its improved version of the MC code. The next step will be performed through the inclusion of the LF spectral function [6].

\section{References}

[1] A. Signori, A. Bacchetta, M. Radici, Int. J. Mod. Phys. Conf. Ser. 25, 9 (2014)

[2] Cates G. et al, E12-09-018, JLAB approved experiment, hallaweb.jlab.org/collab/ PAC/PAC38//E12-09-018-SIDIS.pdf; X. Jiang et al., www.jlab.org/exp prog/ generated/apphalla.html; J. Arrington et al., www.jlab.org/ jinhuang/12GeV/12GeV LongitudinalHe3.pdf

[3] C. Ciofi degli Atti, E. Pace and G. Salmè, Physical Review C 46, R1591 (1992)

[4] C. Ciofi degli Atti, E. Pace, G. Salmè and S. Scopetta, Physical Review C 48, R968 (1993)

[5] S. Scopetta, Physical Review D 75, 10 (2007)

[6] A. Del Dotto, L.P. Kaptari, E. Pace, G. Salmè and S. Scopetta, to be published

[7] A. Del Dotto, E. Pace, G. Salmè and S. Scopetta, Il Nuovo Cimento C, 35, 101-106 (2012)

[8] E. Pace, G. Salmè, S. Scopetta, A. Del Dotto and M. Rinaldi, Few-Body Systems, 54, 1079-1082 (2013)

[9] L.P. Kaptari, A. Del Dotto, E. Pace, G. Salmè and S. Scopetta, arXiv: 1307 . 2848

[10] C. Ciofi degli Atti and L. P. Kaptari, Physical Review C 83, 8 (2011) 